\title{
Computational hemodynamic analysis of the offending vertebral artery at the site of neurovascular contact in a case of hemifacial spasm associated with subclavian steal syndrome: illustrative case
}

\author{
Keita Tominaga, MD, ${ }^{1}$ Hidenori Endo, MD, PhD, ${ }^{1,2,3}$ Shin-ichiro Sugiyama, MD, $\mathrm{PhD},{ }^{4}$ Shin-ichiro Osawa, MD, PhD, ${ }^{1}$ \\ Kuniyasu Niizuma, MD, PhD, ${ }^{1,5,6}$ and Teiji Tominaga, MD, $\mathrm{PhD}^{1}$
}

\begin{abstract}
Departments of ${ }^{1}$ Neurosurgery and ${ }^{6}$ Neurosurgical Engineering and Translational Neuroscience and ${ }^{3}$ Division of Advanced Cerebrovascular Surgery, Tohoku University Graduate School of Medicine, Sendai, Japan; ${ }^{2}$ Department of Neurosurgery, Kohnan Hospital, Sendai, Japan; ${ }^{4}$ Department of Neuroanesthesia, Kohnan Hospital, Sendai, Japan; and ${ }^{5}$ Department of Neurosurgical Engineering and Translational Neuroscience, Graduate School of Biomedical Engineering, Tohoku University, Sendai, Japan
\end{abstract}

\begin{abstract}
BACKGROUND Hemifacial spasm (HFS) is caused by neurovascular contact along the facial nerve's root exit zone (REZ). The authors report a rare HFS case that was associated with ipsilateral subclavian steal syndrome (SSS).

OBSERVATIONS A 42-year-old man with right-sided aortic arch presented with progressing left HFS, which was associated with ipsilateral SSS due to severe stenosis of the left brachiocephalic trunk. Magnetic resonance imaging showed contact between the left REZ and vertebral artery (VA), which had shifted to the left. The authors speculated that the severe stenosis at the left brachiocephalic trunk resulted in the left VA's deviation, which was the underlying cause of the HFS. The authors performed percutaneous angioplasty (PTA) to dilate the left brachiocephalic trunk. Ischemic symptoms of the left arm improved after PTA, but the HFS remained unchanged. A computational fluid dynamics study showed that the high wall shear stress (WSS) around the site of neurovascular contact decreased after PTA. In contrast, pressure at the point of neurovascular contact increased after PTA.
\end{abstract}

LESSONS SSS is rarely associated with HFS. Endovascular treatment for SSS reduced WSS of the neurovascular contact but increased theoretical pressure of the neurovascular contact. Physical release of the neurovascular contact is the best treatment option for HFS.

https://thejns.org/doi/abs/10.3171/CASE21447

KEYWORDS hemifacial spasm; subclavian steal syndrome; computational fluid dynamics; endovascular treatment; microvascular decompression

Neurovascular compression syndromes such as hemifacial spasm (HFS) and trigeminal neuralgia (TN) are generally caused by neurovascular contact between the vascular structure and the cranial nerves. In HFS, neurovascular compression occurs along the root exit zone (REZ) of the facial nerve, most commonly by either the anterior inferior cerebellar artery or the posterior inferior cerebellar artery (PICA) and rarely by vertebral artery (VA). Microvascular decompression (MVD) is an effective microsurgical treatment option for HFS through releasing the contact of vessels from the nerves. ${ }^{1}$ In contrast, endovascular treatment is rarely effective when the offending structure is a cerebral aneurysm. ${ }^{2-7}$ Here, we report a rare case with progressing HFS, which was associated with subclavian steal syndrome (SSS). We attempted to cure both pathologies by an endovascular approach, which was not successful for the HFS but only for the SSS. We validated the result of the endovascular treatment using a computational fluid dynamics (CFD) study.

\section{Illustrative Case}

A 42-year-old male presented with progressing left HFS, which was refractory to any medical treatments. He had a history of asymptomatic right-sided aortic arch, in which the left brachiocephalic trunk arose first, followed by the right common carotid and

ABBREVIATIONS 3D = three dimensional; BA = basilar artery; CFD = computational fluid dynamics; DSA = digital subtraction angiography; HFS = hemifacial spasm; $\mathrm{MRI}=$ magnetic resonance imaging; MVD = microvascular decompression; $\mathrm{PICA}=$ posterior inferior cerebellar artery; REZ = root exit zone; SSS = subclavian steal syndrome; TN = trigeminal neuralgia; VA = vertebral artery; WSS = wall shear stress. INCLUDE WHEN CITING Published September 20, 2021; DOI: 10.3171/CASE21447.

SUBMITTED August 8, 2021. ACCEPTED August 11, 2021.

(C) 2021 The authors, CC BY-NC-ND 4.0 (http://creativecommons.org/licenses/by-nc-nd/4.0/). 

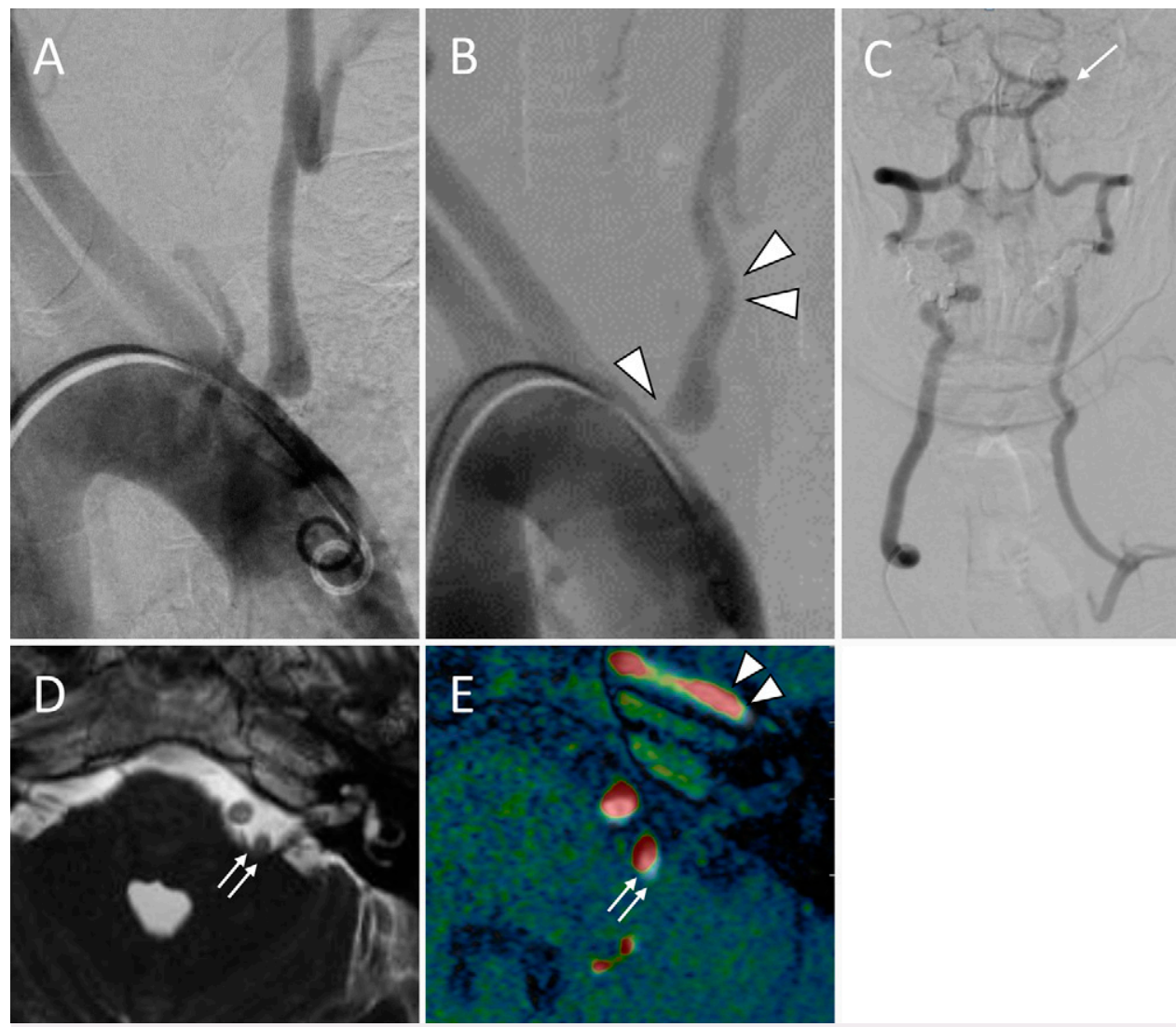

FIG. 1. A: Initial aortography showing stenosis at the left brachiocephalic trunk. Note that the patient had right-sided aortic arch, in which the left brachiocephalic trunk arose first, followed by the right common carotid and right subclavian arteries. B: Follow-up angiography showing progression of the stenosis (arrowhead) and redundancy of the left brachiocephalic trunk (double arrowheads). C: Right vertebral angiography showing retrograde filling of the left VA through vertebrobasilar junction, which was abnormally deviated to the left side (arrow). D: Heavily T2-weighted imaging showing the contact between the left VA and the REZ of the left facial nerve (double arrows). E: Fusion MRI showing the progression of the neurovascular contact in the preoperative status (white) and in the previous status (red) (double arrows). Double arrowheads show the unchanged location of the left internal carotid artery, which was used as control of vessel position.

right subclavian arteries. Digital subtraction angiography (DSA) performed 1 year before the onset of HFS showed stenosis at the origin of the left brachiocephalic trunk, which caused left SSS (Fig. 1A). A second DSA performed after the onset of HFS showed aggravation of the stenosis and redundancy of the left brachiocephalic trunk (Fig. 1B). Right vertebral angiography showed retrograde filling of the left VA through the vertebrobasilar junction, which was abnormally deviated to the left side (Fig. 1C). Magnetic resonance imaging (MRI) showed contact between the left VA and the REZ of the left facial nerve (Fig. 1D), which had progressed compared with the previous MRI finding (Fig. 1E).

In addition to the left HFS, he suffered from ischemic symptoms of the left arm. Blood pressure measured in the left arm (102/81 $\mathrm{mmHg}$ ) was lower than that in the right arm $(136 / 74 \mathrm{mmHg})$. We speculated that aggravation of the stenosis at the left brachiocephalic trunk may be an underlying cause of not only SSS but HFS. We performed percutaneous balloon angioplasty of the left brachiocephalic trunk (Fig. 2A). Ischemic symptoms of the left arm resolved, and the blood pressure measured the following day after treatment in the left and right arm was $134 / 95$ and $131 / 88 \mathrm{mmHg}$, respectively. However, the left HFS remained unchanged after endovascular treatment. Six months after endovascular treatment, we performed MVD, and the symptom disappeared after surgery.

We conducted CFD analysis for the quantitative examination of the periprocedural hemodynamic status. Detailed information is described in Supplementary Methods. We reconstructed the vascular system consisted of bilateral VAs and a basilar artery (BA) from the three-dimensional (3D) data of computed tomography angiography (Fig. 2B). The mean blood flow rate in the $B A(Q)$ was estimated using a following formula: ${ }^{8} \mathrm{Q}=\pi \tau \mathrm{D}^{3} / 32 \mu$. $\mathrm{Q}, \tau, \mu$, and $\mathrm{D}$ denote the blood flow rate, wall shear stress (WSS), fluid viscosity, and vascular diameter, respectively.

We assumed that the mass flow rate in the BA was maintained due to the autoregulation of cerebral blood flow (Fig. 2B). In addition, we substituted mean values of the blood pressure for inlet and outlet boundaries (Fig. 2B) based on the speculation as follows. For pretreatment status, the stenosis at the origin of the left brachiocephalic trunk caused a drop in pressure in the left VA. The resulting pressure difference from the right VA to the left VA drove retrograde blood flow in the left VA. For posttreatment status, the release of 


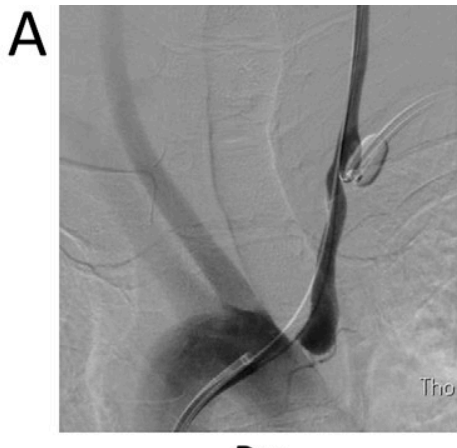

Pre

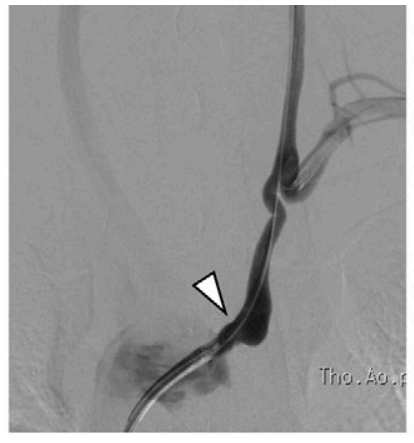

Post
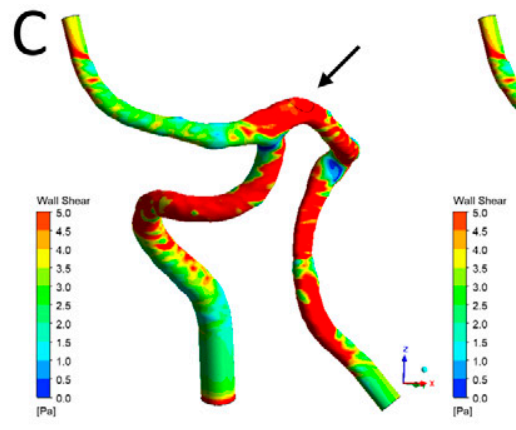

Pre

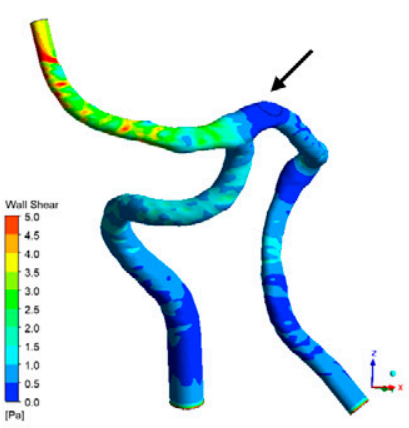

Post

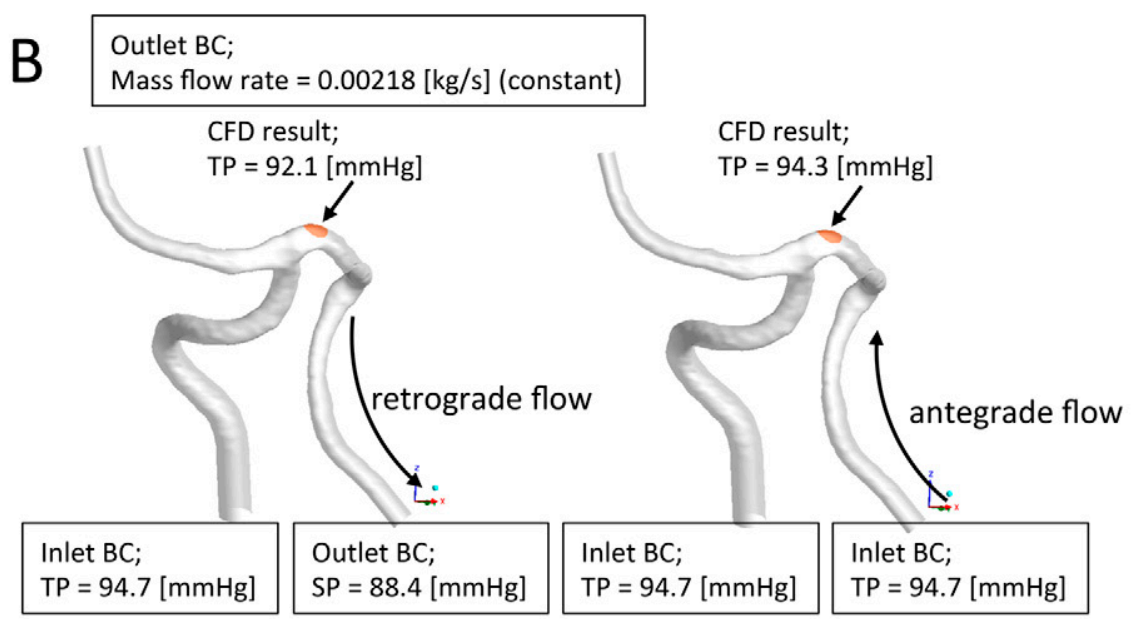

Pre

Post

FIG. 2. A: Pre- and postoperative angiography showing the resolution of stenosis at the left brachiocephalic trunk (arrowhead). B: Models for CFD. Boundary conditions used in CFD simulation are also presented. The results of the CFD analysis revealed that total pressure at the neurovascular contact (orange) increased after treatment. BC = boundary condition; SP = static pressure; TP = total pressure. C: Contour maps of WSS magnitude. Mean values of WSS magnitude in vascular contact (arrow) are 7.33 and $0.27 \mathrm{~Pa}$ in pre- and posttreatment status, respectively.

the stenosis resolved the pressure difference, which restored antegrade blood flow in the left VA.

Following the conventions for CFD in large arteries, the blood was treated as an incompressible Newtonian fluid (density: $1,050 \mathrm{~kg} / \mathrm{m}^{3}$; viscosity: $0.004 \mathrm{~Pa} \cdot \mathrm{s}$ ), vessel walls were assumed to be rigid, and noslip boundary conditions were applied on the walls. ${ }^{9,10}$ A finite-volume method package, ANSYS 14.5 (ANSYS, Inc.), was used to solve the governing equations, which included the 3D Navier-Stokes equations and the equation of continuity.

In the post-CFD analysis, we examined the mean values of WSS magnitude and total pressure in the neurovascular contact. Under the pretreatment hemodynamics, the impact of retrograde blood flow on the neurovascular contact caused a focal elevation of the WSS magnitude (mean: 7.33 Pa). We found that the WSS magnitude at the neurovascular contact decreased under posttreatment antegrade blood flow (mean: $0.27 \mathrm{~Pa}$ ) (Fig. 2C). In contrast, the total pressure at the neurovascular contact increased after treatment (mean: $94.3 \mathrm{mmHg}$ ) (Fig. 2B).

\section{Discussion}

\section{Previous Reports of Endovascular Treatment for HFS}

HFS is a disorder characterized by paroxysmal and involuntary twitching of facial muscles, which is triggered by neurovascular contact between the facial nerve and the blood vessels. MVD is usually effective in releasing this contact, normalizing the function of the affected facial nerve. ${ }^{1,11}$ Endovascular treatment is rarely effective for HFS when the offending arterial structure is cerebral aneurysm. $^{2-7}$ Six cases with HFS caused by VA aneurysms were treated by endovascular treatment in the literature. ${ }^{2-7}$ Parent artery occlusion, intraaneurysmal coil embolization, and flow diverter stent were performed for four fusiform/dissecting aneurysms, one VAPICA aneurysm, and one VA saccular aneurysm, respectively. In these six cases, endovascular material was directly placed at the offending intracranial vascular structure, and HFS was resolved in all cases. One plausible explanation for the resolution of HFS after the endovascular treatment is that pulsatility rather than compression is the stronger pathologic driver in $\mathrm{HFS}^{4}$

\section{Observations}

Endovascular treatment was not effective in improving HFS in the present case. We performed endovascular treatment mainly for the resolution of SSS. We expected that improvement of the brachiocephalic trunk stenosis would have some positive effect on the HFS by normalizing the reversed flow of the left VA. We investigated the causes of failure of this treatment strategy using CFD 
analysis. Satoh et al. investigated the WSS of offending vessels at the neurovascular contact in patients with TN and HFS by conducting a CFD analysis. ${ }^{12}$ They found that some patterns of the changes in the preoperative WSS along the offending vessels may correlate with the neurovascular contact region, which provided useful information for MVD. ${ }^{12}$ In the present study, the magnitudes of the WSS increased around the site of neurovascular contact, which was similar to the results of the previous study. ${ }^{12}$ This increased WSS around the neurovascular contact decreased after the endovascular treatment, which did not affect HFS. Thus, preoperative WSS can be useful to predict the exact region of neurovascular contact, but WSS itself is not associated with the underlying pathology of HFS. In contrast, theoretical pressure of the neurovascular contact calculated by CFD analysis increased after endovascular treatment. Thus, pressure at the neurovascular contact may be associated with the relief of symptoms. Physical release of the neurovascular contact by MVD is the best treatment option to decrease the pressure of neurovascular contact and improve HFS.

\section{Lessons}

SSS is rarely associated with HFS. Endovascular treatment for SSS reduced WSS of the neurovascular contact, which did not affect HFS. In contrast, theoretical pressure of the neurovascular contact calculated by CFD analysis increased after endovascular treatment. Physical release of the neurovascular contact by MVD is the best treatment option to improve HFS.

\section{References}

1. Jannetta PJ, Abbasy M, Maroon JC, Ramos FM, Albin MS. Etiology and definitive microsurgical treatment of hemifacial spasm. Operative techniques and results in 47 patients. J Neurosurg. 1977;47(3): 321-328.

2. Nakagawa I, Takayama K, Kurokawa S, et al. Hemifacial spasm due to contralateral aneurysmal compression of the facial nerve successfully treated with endovascular coil embolization: case report. Neurosurgery. 2011;69(3):E768-E772.

3. Arisawa K, Ochi T, Goto Y, Nanbu S, Shojima M, Maeda K. Coil embolization of VA-PICA aneurysm presenting with hemifacial spasm with assistance of abnormal muscle response monitoring. Journal of Neuroendovascular Therapy. 2020;14(4):146-150.

4. Santiago-Dieppa DR, McDonald MA, Brandel MG, Rennert RC, Khalessi AA, Olson SE. Endovascular flow diversion for hemifacial spasm induced by a vertebral artery aneurysm: first experience. Oper Neurosurg (Hagerstown). 2019;17(3):E115-E118.

5. Sato K, Ezura M, Takahashi A, Yoshimoto T. Fusiform aneurysm of the vertebral artery presenting hemifacial spasm treated by intravascular embolization: case report. Surg Neurol. 2001;56(1):52-55.
6. Nagashima H, Orz Y, Okudera H, Kobayashi S, Ichinose Y. Remission of hemifacial spasm after proximal occlusion of vertebrobasilar dissecting aneurysm with coils: case report. J Clin Neurosci. 2001;8(1):43-45.

7. Kugai M, Suyama T, Kitano M, Hasegawa $H$, Tominaga $Y$, Tominaga $\mathrm{S}$. A case of high-grade arteriovenous malformation manifesting as trigeminal neuralgia successfully treated by embolization in multimodal treatment. J Neuroendovasc Ther. 2019;13(9):382-387.

8. Zarins CK, Zatina MA, Giddens DP, Ku DN, Glagov S. Shear stress regulation of artery lumen diameter in experimental atherogenesis. J Vasc Surg. 1987;5(3):413-420.

9. Steinman DA, Vorp DA, Ethier CR. Computational modeling of arterial biomechanics: insights into pathogenesis and treatment of vascular disease. J Vasc Surg. 2003;37(5):1118-1128.

10. Sugiyama S, Niizuma K, Sato K, et al. Blood flow into basilar tip aneurysms: a predictor for recanalization after coil embolization. Stroke. 2016;47(10):2541-2547.

11. Hyun SJ, Kong DS, Park K. Microvascular decompression for treating hemifacial spasm: lessons learned from a prospective study of 1,174 operations. Neurosurg Rev. 2010;33(3):325-334.

12. Satoh T, Yagi T, Onoda K, et al. Hemodynamic features of offending vessels at neurovascular contact in patients with trigeminal neuralgia and hemifacial spasm. J Neurosurg. 2019;130(6):1870-1876.

\section{Disclosures}

The authors report no conflict of interest concerning the materials or methods used in this study or the findings specified in this paper.

\section{Author Contributions}

Conception and design: Endo, Tominaga. Acquisition of data: Endo, Tominaga, Osawa, Niizuma. Analysis and interpretation of data: Endo, Tominaga, Sugiyama, Niizuma. Drafting the article: Endo, Tominaga. Critically revising the article: Endo, Osawa. Reviewed submitted version of manuscript: Endo, Osawa, Niizuma, Tominaga. Approved the final version of the manuscript on behalf of all authors: Endo. Study supervision: Tominaga.

\section{Supplemental Information}

Online-Only Content

Supplemental material is available with the online version of the article. Supplementary Methods. https://thejns.org/doi/suppl/10.3171/CASE21447.

\section{Correspondence}

Hidenori Endo: Kohnan Hospital, Sendai, Japan. h-endo@nsg.med. tohoku.ac.jp. 